\title{
MULTIPLICATIVE PRIMENESS OF COMPLEXIFICATION FOR REAL ALGEBRAS
}

\author{
Amir A. Mohammed \& $\quad$ Baida S.Abdullah \\ Department of Mathematics Department of Mathematics \\ College of Education $\quad$ College of Education \\ University of Mosul University of Mosul \\ Mosul-Iraq $\quad$ Mosul-Iraq
}

Received

Accepted

2006/6/6

$2006 / 2 / 26$

الملخص

نطلق على أي جبر A بالمضروب أوليا إذا كان كل من A و M (جبر المضروبات

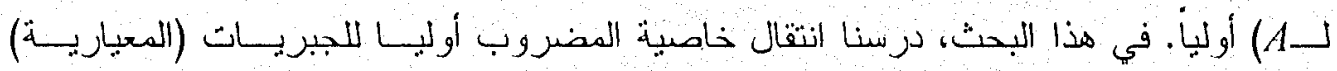

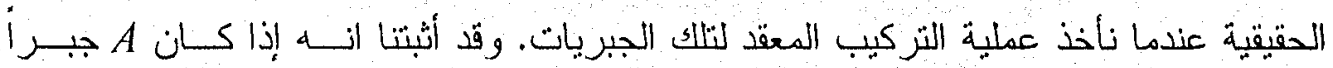

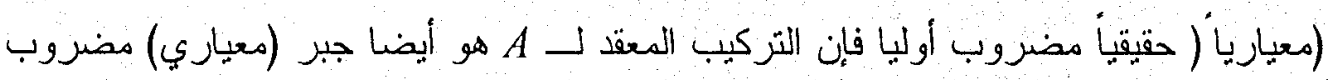
أولياً.

\section{ABSTRACT}

We say that an algebra $A$ is multiplicatively prime if both $A$ and $M(A)$ (the multiplication algebra of $A$ ) are prime. In this paper, we study the transitivity of the property of multiplicative primeness for real (normed) algebra when one take the process of the complexification of such real algebra. We prove that if $A$ is a real (normed) multiplicatively prime algebra, then the complexification of $A$ is also multiplicatively prime(normed) algebra. 


\section{INTRODUCTION AND PRELIMINARIES.}

Throughout this paper $\mathrm{R}, \mathrm{C}$ will denote the real and complex fields respectively. A systemic study for multiplicatively prime algebra was given in [2] and [4] by Cabrera and Mohammed and later these algebras has been extended to multiplicatively semiprime algebras again by Cabrera and Mohammed see [3].There is an interesting (resent) papers related to multiplicatively (semi) prime algebras which explained the connection between these algebras and another important nonassociative algebra like Jordan and Lie algebras see ([1],[5] and [7] ). In this paper, we study the complexification of real multiplicatively prime algebras and we apply our result in the case when algebra equipped with an algebra norm.

We begain by summarizing some definitions and fundemental concepts. An algebra $A$ is said to be semiprime if 0 is the unique ideal $U$ of $A$ with $U^{2}=0$. We also recall that an algebra $A$ is said to be prime if for ideals $U$ and $V$ of $A$ with $U V=0$ implies either $U=0$ or $V=0$. The multiplication algebra $M(A)$ of an algebra $A$ is defined as the subalgebra of $L(A)$ (the algebra of all linear operators on $A$ ) generated by the identity operator $I d_{A}$ and the set $\left\{L_{a}, R_{a}: a \in A\right\}$ where $L_{a}$ and $R_{a}$ will mean the operators of left and right (respectively) multiplication by $a$ on $A$. An algebra $A$ is said to be multiplicatively prime if both $A$ and $M(A)$ are prime.

The concept of extended centroid was introduced and developed first by W. S. Martindale in the case of prime associative rings, and later this concept has been extended to the more general context of prime nonassociative algebras See [8]. Let $A$ be a semiprime algebra. A partially defined centralizer on $A$ is a linear mapping $f: \operatorname{dom}(f) \rightarrow A$, whose 
domain is a nonzero ideal of $A$, and such that $f(a x)=a f(x)$ and $f(x a)=f(x) a$ for all $a$ in $A$ and all $x$ in $\operatorname{dom}(f)$. If $\operatorname{dom}(f)$ is an essential ideal of $A$, then $f$ will be called essentially defined centralizer on $A$. The set $C(A)$ of all equivalence classes $[f]$ under the operations induced by addition and composition of partially defined operators is a Von Neumann regular commutative ring called the extended centroid of $A$. Moreover $C(A)$ is a field if, and only if, $A$ is prime.

In section 2 we prove that if $A$ is centrally closed multiplicatively prime real algebra, then the complexification of $A$ is also centrally closed multiplicatively prime algebra. Furthermore the mapping from the complexification of multiplication algebra of $A$ into the multiplication algebra of the complexification of $A$ is a $C$-algebra isomorphism. While in section 3 we prove if $(A,\|\|$,$) is a centrally closed multiplicatively$ prime real normed algebra, then the complexifcation of $A$ is also centrally closed multiplicatively prime normed algebra. Furthermore the mapping from the complexification of mulliplication algebra of $A$ into multiplication algebra of the complexification of $A$ is a topological.

\section{COMPLEXIFICATION OF REAL MULTIPLICATIVE-}

\section{LY PRIME ALGEBRAS.}

In this section, we study the refect of the multiplicative primeness property in real algebra whenever taking the process of the complexification of such real algebra, for more details for the complexification of real algebra, see [6], [8] and [9]. Let $A$ be a real algebra and let us denote by $M(A)_{C}$ the complexification of the multiplication algebra of $A$ and $M\left(A_{C}\right)$ be the multiplication algebra of the complexification of $A$. We begin by the first main result: 
Theorem 1. Let A be centrally closed multiplicatively prime real algebra. Then the complexification of $A$ is also centrally closed multiplicatively prime algebra. Furthermore the mapping from the complexification of multiplication algebra of $A$ into the multiplication algebra of the complexification of $A$ is a C-algebra isomorphism.

Proof. Let $\phi$ be a mapping from $M(A)_{C}$ into $M\left(A_{C}\right)$ defined by:

$$
\phi(F+i G)(a+i b)=((F(a)-G(b))+i(F(b)+G(a)),
$$

for all $(F+i G) \in M(A)_{C}$ and for all $(a+i b) \in A_{C}$.

Clearly $\phi$ is well-define. Now we shall prove that the mapping $\phi$ is isomorphism. One can check easily that:

(i) $\phi\left((F+i G)+\left(F^{\prime}+i G^{\prime}\right)\right)(a+i b)=\phi(F+i G)(a+i b)+\phi\left(F^{\prime}+i G^{\prime}\right)(a+i b)$

(ii) $\phi((\alpha+i \beta)(F+i G))(a+i b)=(\alpha+i \beta)[\phi(F+i G)(a+i b)]$

and

$(i i i) \phi\left((F+i G)\left(F^{\prime}+i G^{\prime}\right)\right)(a+i b)=\left[\phi(F+i G) \phi\left(F^{\prime}+i G^{\prime}\right)\right](a+i b)$

for all $\left(F^{\prime}+i G\right),\left(F^{\prime}+i G^{\prime}\right) \in M(A)_{1}$ and for all $(a+i b) \in A_{C}$

As consequence $\phi$ is $C$-algebra homomorphism. To see that $\phi$ is injective, let $\phi(F+i G)=0$ this implies that:

$$
\phi(F+i G)(a+i b)=0 \text { for all }(a+i b) \in A_{c},
$$

In particular $0=\phi(F+i G)(a)=F(a)+i G(a)$

$F(a)=0$ and $G(a)=0$ for all $a \in A$, therefore $F=0$ and $G=0$.

It is still for us to prove that $\phi$ is surjective. Since $\phi$ is a homomorphism, then we have that $\phi\left(M(A)_{C}\right)$ is a subalgebra of $M\left(A_{C}\right)$. But this subalgebra contain the genereaters of $M\left(A_{C}\right)$ because: $I d_{A_{C}}=\phi\left(I d_{A}+i O\right)$ 


$$
\begin{aligned}
\phi\left(L_{x}+i L_{y}\right)(a+i b)= & L_{x}(a)-L_{y}(b)+i\left[L_{x}(b)+L_{y}(a)\right] \\
& =x a-y b+i(x b+y a) \\
& =(x+i y)(a+i b) \\
L_{x+i y}^{A_{C}}=\phi\left(L_{x}+i L_{y}\right) & =L_{x+y y}^{A_{c}}(a+i b)
\end{aligned}
$$

Similary,

$R_{x+i y}^{A_{c}}=\phi\left(R_{x}+i R_{y}\right)$

Therefore, $\phi\left(M(A)_{C}\right)=M\left(A_{C}\right)$. As cosequence $\phi$ is surjective.

Now we shall prove that $M\left(A_{C}\right)$ is a closed prime complex algebra. Since $M(A)$ is a real prime algebra with $C(A)=R$. By [6] we have $C(A) \cong C(M(A))$ this implies that $C(M(A))=R$. As a consequence, $M(A)$ is centrally closed.

We apply [8, Theorem 3.5] to get $M(A) \otimes_{R} C \cong M(A)_{\text {, }}$ is a closed prime algebra. But from the proof of the first part of our theorem 1 we conclued that $M(A)_{1} \equiv M\left(A_{1}\right)$. Therefore $M\left(A_{C^{\prime}}\right)$ is a closed prime complex algebra. Since $A$ is centrally prime algebra, it follows that by 18 , Theorem 3.5] $A_{c}$. is also closed prime complex algebra. As a consequence $A_{C}$ is multiplicatively prime algebra. 1

Remark 1. Let $A$ be an algebra. $A$ is prime if, and only if, $C(A)$ is a field see [8], it follows that the condition of primeness in the theorem 1 could be usefull. While, if $A$ is semiprime, then $C(A)$ is a Von Neumann regular commutative ring see [8]. So, one can not get the same result at theorem 1 when $A$ is semiprime.

\section{APPLICATION TO NORMED ALGEBRAS.}

Our aim now is to answer the following equation:

Is the isomorphism at theorem 1 is topological when ever $A$ is equiped with algebra norm? 
The answer is true by the next theorem. Recall that, a normed algebra is an algebra $A$ with a norm \|\|$: A \rightarrow R^{+}$satisfying $\|a b\| \leq\|a\|\|b\|$ for all $a, b \in A$. Also, recall that an isomorphism $\phi$ from a normed algebra $A$ into a normed algebra $B$ is said to be topological if, and only if, $\phi$ and $\phi^{-1}$ is continuous.

Theorem 2. Let $(A,\|\|)$ be centrally closed multiplicatively prime real normed algebra. Then the complexification of $A$ is also centrally closed multiplicatively prime normed algebra. Furthermore the mapping from the complexification of normed multiplication algebra of $A$ into the normed multiplication algebra of the complexification of $A$ is a topological.

Proof. Let us denote by $A_{c}$, be the complexification of $A$. By theorem 1, $A_{1}$ is a centrally closed multiplicatively prime algebra. If $A$ is normed real algebra, then by ([9], theorem 1.3.2) we have that $A_{c}$ is a normed complex algebra with a norm \|\|$_{c}$ defined on $A_{c}$ by:

$$
\begin{aligned}
& \|(a, b)\|_{C}=\frac{1}{\sqrt{2}} \operatorname{Sup}\left|e^{i \theta}(a, b)\right| \\
& \forall(a, b) \in A_{C} \equiv(A+i A) \text {. Where }|(a, b)|=\|a\|+\|b\|
\end{aligned}
$$

Note that, \|\|$\|_{C}$ is equivelent to the norm \|\|\|\| defined by $\|(a, b)\|=\max \{\|a\|,\|b\|\}$.

Now, define $\phi$ from $M(A)_{C}$ into $M\left(A_{C}\right)$ as in theorem 1 . We will use the following diagram: 

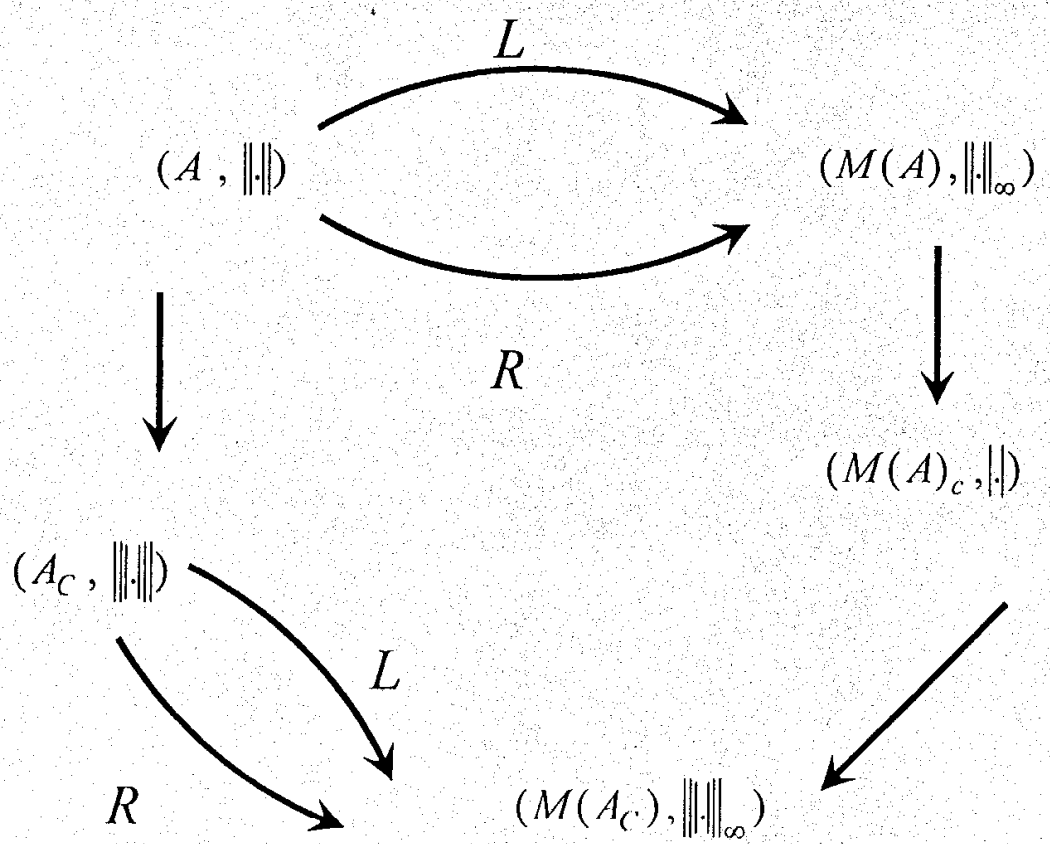

$\left(M\left(A_{C}\right),\|\|_{\infty}\right)$

To prove $\phi$ is topological first we will prove that

$$
\phi:\left(M(A)_{C}, \|\right) \rightarrow\left(M\left(A_{C}\right),\|\cdot\|_{\infty}\right)
$$

is continuous, that is, $\|\phi(F+i G)\|_{\infty} \leq k|F+i G|$,

for some positive constant $k>0$ and all $F+i G \in M(A)_{c}$ (where $F, G \in M(A))$. For $a+i b \in A_{C}$ (where $a, b \in A$ ), we have

$$
\begin{aligned}
& \|\phi(F+i G)(a+i b)\|=\|F(a)-G(b)+i(G(a)+F(b))\| \\
& \leq 2 \operatorname{Max}\{\|F(a)-G(b)\|,\|G(a)+F(b)\|\} \\
& \leq 2 \operatorname{Max}\left\{\|F\|_{\infty}\|a\|+\|G\|_{\infty}\|b\|,\|G\|_{\infty}\|a\|+\|F\|_{\infty}\|b\|\right\} \\
& \leq 2 \operatorname{Max}\{\|a\|,\|b\|\}\left(\|F\|_{\infty}+\|G\|_{\infty}\right) \\
& \leq 2\left(\|F\|_{\infty}+\|G\|_{\infty}\right)\|a+i b\|
\end{aligned}
$$

Now, take the supremum in above inequality over $A_{c}$, we have: 


$$
\begin{aligned}
\|\phi(F+i G)\|_{\infty} & \leq 2\left(\|F\|_{\infty}+\|G\|_{\infty}\right) \\
& \leq 4 M a x\left\{\|F\|_{\infty},\|G\|_{\infty}\right\} \\
& \leq 4|F+i G| .
\end{aligned}
$$

This proves that $\phi$ is continuous. Finally, we will prove that $\phi^{-1}$ is continuous. Note that $\phi^{-1}$ is given by:

$$
\phi^{-1}:\left(M\left(A_{C}\right),\|.\|_{\infty}\right) \rightarrow\left(M(A)_{C}, .\right),
$$

We shall prove that $\left|\phi^{-1}(F+i G)\right| \leq k\|\mid F+i G\|_{\infty}$,

for some positive constant $k$ and for all $F, G \in M(A)$.

This is equivalent to

$$
|F+i G| \leq k\|\phi(F+i G)\|_{\infty}
$$

Now, for all $a \in A$ we have

$$
\begin{aligned}
& \operatorname{Max}\{\|F(a)\|,\|G(a)\|\} \leq\|F(a)+i G(a)\| \\
& =\|\phi(F+i G)(a+i 0)\| \\
& \leq\|\phi(F+i G)\|_{\infty}\|(a+i 0)\| \\
& =\|\phi(F+i G)\|_{\infty}\|(a)\|
\end{aligned}
$$

Now, take the supremum in above inequality over $A$ with $\|d\|=1$, we have

$$
\frac{1}{2}|F+i G| \leq \operatorname{Max}\left\{\mid F\left\|_{\infty},\right\| G \|_{\infty}\right\} \leq\|\phi(F+i G)\|_{\infty} .
$$

As a consequence, $\phi^{-1}$ is continuous. Therefore, $\phi$ is a toplogical. ${ }^{1}$

\section{REFERENCES}

1. M. Cabrera and J. C. Cabello. (2004). Structure theory for multiplicatively semiprime algebras. Journal of Algebra 282, 386421.

2. M. Cabrera, A. A. Mohmmed (1999). Extended centroid and central closure of the multiplication on algebra. Comm. Algebra 27(12): 5723-5736. 
3. M. Cabrera and A. A. Mohmmed (2001). Extended centroid and centeal closure of multiplicatively semiprime algebras. Comm. algebra $29(3): 1215-1233$.

4. M. Cabrera and A. A. Mohmmed (2002). Totally multiplicatively prime algebras. Proc. Royal Soc. Edinburg 132 A: $1145-1162$.

5. M. Cabrera, J. C. Cabello, G. Lopez and W. S. Martindale III. (2004). Multiplicative semiprimeness of skew Lie Algebras. Communications in algebra, Vol. 32 , No. 9, pp. 3487-3501.

6. M. Cabrera and A. Rodrignez. (1992). Nonassociative ultraprime normed algebras. Quart. J. Math Oxford (2) 43,1-7.

7. M. Cabrera and A. R. Villena. Multiplicative semiprimeness of Nondegenerate Jordan Algebras. (2004). Communications in algebra, Vol. 32 , No. 10, pp. 3995-4003.

8. T. S. Erickson, W. S. Martindale III and J. M. Osborn. (1975). Prime nonassociative algebras pacific. J. Math. 60, 49-63.

9. C. E. Rickart. (1960). General theory of Banach algebras. New York, Van Nostrand. 